\title{
One Laptop per Child: Análise sobre as implementações no Brasil e no Uruguai
}

\section{Avelino Ferreira Gomes Filho ${ }^{1}$, Flávio Duarte ${ }^{1}$, Josemary Corrêa Kopke ${ }^{1}$, Fábio Ferrentini Sampaio ${ }^{2}$}

${ }^{1}$ Programa de Pós-Graduação em Informática

Universidade Federal do Rio de Janeiro (UFRJ)

${ }^{2}$ Programa de Pós-Graduação em Informática

Instituto Tércio Pacitti de Aplicações e Pesquisas Computacionais

Universidade Federal do Rio de Janeiro (UFRJ)

avelino.filho@ppgi.ufrj.br, \{flavioduarte, jokopke\}@ufrj.br, ffs@nce.ufrj.br

\begin{abstract}
The One Laptop per Child (OLPC) is a model that aims to transform the learning process of every child in developing countries through Information and Communications Technology (ICT). However, cultural, economic and social diversities make each model implementation unique. This study analyses the application of OLPC in Uruguay and Brazil. It aims to understand and describe common aspects that are important to the success of this kind of implementation. Our goal is to contribute with future projects that use ICT to transform the way that children learn.
\end{abstract}

Resumo. O One Laptop per Child (OLPC) é uma proposta cujo objetivo busca, através do uso da Tecnologia de Informação e Comunicação (TIC), transformar o processo de aprendizagem de todas as crianças em países de desenvolvimento. Entretanto, as diversidades culturais, econômicas e sociais fazem com que cada implementação seja única. O presente trabalho faz uma análise crítica sobre a aplicação do OLPC no Uruguai e no Brasil. Procura evidenciar aspectos comuns que sejam importantes para aplicação dessa proposta. Com isso, esperamos contribuir com novos projetos que objetivem transformar o processo de ensino e aprendizagem através do uso das TICs.

\section{Introdução}

Ao longo dos anos, diferentes iniciativas surgiram com o intuito de tornar o computador uma ferramenta pedagógica que auxiliasse alunos e professores no processo de ensino e aprendizado. Busca-se o uso das TIC para criar modelos criativos e estimulantes que possibilitem transformar a forma como os alunos desenvolvem conhecimento [Alves et al. 2015].

Em 2005, uma dessas iniciativas foi apresentada no Fórum Econômico Mundial Econômico de Davos. Nicolas Negroponte descreveu a proposta One Laptop per Child (OLPC) cujo objetivo era entregar um laptop de baixo custo conectado à internet para cada criança no planeta [Kraemer et al. 2009]. Essa proposta nasce a partir de uma abordagem construcionista [Papert 1993] em que o computador não é apenas uma ferramenta substitutiva do quadro-negro, lápis e caderno e sim, algo capaz de alterar a forma como o processo de ensino é realizado [Canal and Almeida 2010, Cysneiros et al. 2011]. 
Em 2007, tanto o Brasil quanto o Uruguai implementaram instâncias do OLPC. A reflexão que propomos para essa pesquisa é, através de um olhar crítico, analisar essas implementações a fim de compreender quais fatores podem contribuir para o sucesso desse tipo de empreitada e quais os desafios devem ser superados. O trabalho está assim organizado: A Seção 2 apresenta os trabalhos relacionados. A Seção 3 apresenta o método utilizado na pesquisa. As seções 4 e 5 descrevem os programas do Uruguai e Brasil respectivamente. Por fim, a Seção 6 apresenta a análise sobre os dados e conclui a pesquisa.

\section{Trabalhos Relacionados}

Por ser uma proposta relevante, o OLPC vem sendo estudado ao longo do tempo por diferentes pesquisadores. Nugroho e Londsdale (2010) apresentam uma revisão da literatura sobre a adoção do OLPC no mundo. Eles oferecem informações bastante resumidas sobre os métodos utilizados na implantação do OLPC em 20 países, incluindo o Brasil e o Uruguai. Apresentam informações sintéticas sobre os computadores utilizados e avaliações preliminares.

Com foco próximo à este artigo, os trabalhos de Ferrando et al. (2010) e Melo et al. (2014) avaliam os impactos do Plano Ceibal sobre a educação uruguaia. Eles proporcionam uma relevante discussão sobre os critérios que devem ser adotados para medir o sucesso ou fracasso da implementação neste país.

Os professores Sampaio e Elia (2012) organizaram o livro Projeto Um Computador por Aluno: Pesquisas e perspectivas. Esse livro agrupou mais de 20 estudos elaborados por diferentes pesquisadores sobre o UCA. Pelo fato do Brasil ser um país extenso, ter uma população grande e com disparidades sócio-econômicas-culturais significativas, esse trabalho tem alta relevância por apresentar como o projeto recebeu diferentes abordagens pelo país.

\section{Método de Pesquisa}

O objetivo desse trabalho é analisar as implementações e resultados do Plano Ceibal e do Projeto Um Computador por Aluno (UCA) para evidenciar quais fatores são relevantes para o sucesso desse tipo de empreitada. A pergunta dessa pesquisa é: O que está dando certo e quais são os desafios enfrentados em cada implementação?

A análise realizada buscou evidenciar informações sobre os seguintes critérios: 1) Qual o contexto e objetivos de cada implementação? 2) Quantidade de alunos atendidos; 3) Método de formação dos professores e quantidade de professores formados; 4) Envolvimento da sociedade para apoiar os Programas e meios de integração do projeto; 5) Resultados alcançados.

O método de pesquisa adotado é a Pesquisa Documental. Este tipo de pesquisa tem forte relação com a pesquisa bibliográfica. A diferença está na natureza das informações adquiridas. A pesquisa documental analisa também materiais que ainda não receberam um tratamento analítico prévio, ou que ainda podem ser reelaborados de acordo com os objetivos da pesquisa [Gil 1999]. A pesquisa inicial foi feita nas bases da Scielo, Scopus, IEEE e Google Scholar. Outras informações revelantes proveem de bases governamentais e supranacionais, bem como através de entrevista com pesquisador ligado ao UCA. 


\section{Modelo Uruguaio: Plan Ceibal}

O Uruguai possui uma extensão territorial de $177.414 \mathrm{~km}^{2}$, menor que o estado do Paraná $\left(199.314 \mathrm{~km}^{2}\right)$. Sua população é de 3,4 milhões de habitantes, menor que a população do município do Rio de Janeiro (4 milhões de habitantes). O Índice de Desenvolvimento Humano (IDH) é considerado elevado e 98,4\% da população é alfabetizada [IBGE 2013, MEC 2013, CIA 2015].

No final de 2006, o governo uruguaio lançou o Plan de Conectividad Educativa de Informática Básica para el Aprendizaje en Línea (Plan Ceibal). Esse plano tinha como objetivo fazer com que cada aluno e professor de escola pública recebesse de forma gratuita um computador portátil conectado à internet [Plan Ceibal 2015]. Ele foi institucionalizado em abril de 2007 e se tornou uma política nacional que busca atender não apenas as escolas públicas, como também as escolas privadas e o ensino pré-escolar, fundamental e médio.

Ao longo dos anos o projeto foi aperfeiçoado e hoje possui os seguintes objetivos: Contribuir com o aperfeiçoamento da qualidade da educação mediante a integração das tecnologias utilizadas na sala de aula, centro escolar e núcleo familiar; Promover a igualdade de oportunidade para todos os alunos de educação primária através da distribuição de computadores portáteis para alunos e professores; Desenvolver uma cultura colaborativa em quatro dimensões: aluno-aluno, aluno-professor, professor-professor e aluno-família-escola; Promover a alfabetização e criticidade eletrônica na comunidade pedagógica atendendo aos princípios éticos [Plan Ceibal 2015].

\subsection{Quantidade de alunos e professores atendidos}

Em 2013, o Uruguai entregou o milionésimo computador. Dos 852.999 alunos do préescolar até o ensino médio público e privado, 559.836 alunos (65\%) já são atendidos por computadores e internet oferecidos pelo Plano Ceibal. Com relação aos professores, dos 74.637 professores atuantes, $47.623(64 \%)$ já receberam os computadores do Ceibal. Entretanto, cerca de 400 mil computadores foram inutilizados por avarias ou obsolescência [MEC 2013].

\subsection{Formação dos Professores}

Para trabalhar com as ferramentas de TIC providas pelo Plano Ceibal, os professores possuem diversos meios de formação e treinamento continuado. Na pesquisa, foi possível identificar que há cursos de graduação em licenciatura que buscam ensinar para os futuros docentes o uso de TIC para apoio à educação [Rivoir and Lamschtein 2012]. Não foi possível determinar se há obrigatoriedade para todas as universidades e nem a abordagem utilizada para essa formação.

Além da formação universitária, há diferentes meios de formação complementar para professores. Esses cursos são oferecidos presencialmente em centros do Plano Ceibal ou nas redes de apoio formadas por voluntários. Também estão disponíveis no Portal Ceibal diversos cursos no formato de Ensino a Distância (EAD) [Rivoir and Lamschtein 2012].

\subsection{Meio de Integração}

O Portal Ceibal (http: / / www. ceibal . edu . uy /) é o principal meio de integração do Plano. Além de oferecer cursos, ele também permite o acesso à biblioteca digital 
para alunos e professores, objetos de aprendizagem, jogos educacionais, vídeos sobre temas de disciplinas, informações sobre equipamentos (modelos, aquisição, reparo, troca e descarte). Ademais, possui espaços dedicados à instituição de ensino e aos familiares dos alunos.

\subsection{Envolvimento da Sociedade}

Durante a pesquisa, foi possível perceber algumas iniciativas de apoio ao Plano que partiram da sociedade. Três dessas merecem destaque especial, pois são pilares importantes de fomento e apoio ao Ceibal. São elas: A Red de Apoyo ao Plan Ceibal (RAP Ceibal) é uma rede formada por mais de 800 voluntários espalhada por todo o país. Ela busca auxiliar professores e alunos na utilização de ferramentas de TIC aplicadas à Educação. O Flor de Ceibo é um projeto interdisciplinar promovido pela Universidad de la República que tem como objetivo gerar contribuições acadêmicas que possam aprimorar as práticas utilizadas no Ceibal. Esse projeto tem foco construcionista, conforme descrito por Pappert [Papert 1993], e pretende tornar o uso das TICs como base de apoio para o processo de ensino e não apenas uma ferramenta substitutiva do material físico. Por fim, o Ceibal Jam! (http: / / ceibal jam. org/) é uma rede formada por desenvolvedores de software voluntários que buscam criar meios de colaboração com professores, alunos e instituições de ensino para criar softwares educacionais baseados nos modelos de Software Livre. Além disso, essa rede oferece cursos de desenvolvimento e engenharia de softwares educacionais [Rivoir and Lamschtein 2012].

\subsection{Resultados Alcançados}

É possível perceber que houve uma evolução significativa quanto à universalização do acesso aos recursos tecnológicos. Em 2006, antes do início do plano, 47\% das famílias das classes sociais mais altas tinham acesso à computadores em casa, enquanto apenas 5\% das famílias das classes sociais mais baixas tinham acesso a esse bem. Em 2013, esses números foram para $79 \%$ e $66 \%$ respectivamente. Isso demonstra que o Plano vem sendo bem sucedido no seu propósito primário de universalizar o acesso às TICs [Marandino and Wunnava 2014].

Com relação aos resultados sobre o processo de ensino e aprendizagem, não há unanimidade entre os avaliadores. Para Melo et al. (2013), o Plano não é capaz de apresentar um resultado significativo com relação às disciplinas de matemática e leitura. Já para Ferrando et al. (2010), é necessário analisar as diversas dimensões do Plano e não apenas algumas poucas disciplinas. Para esses autores, os alunos atendidos pelo Ceibal utilizam mais os recursos das TICs do que aqueles que possuem computador, mas ainda não são atendidos pelo Plano. Além disso, os autores ressaltam que os alunos são mais motivados para frequentar as aulas e estudar em casa. Eles concluem que os impactos são positivos. Outros autores destacam que o simples fato de colocar o computador com acesso à internet a disposição de alunos e professores já é capaz de provocar uma mudança no processo de ensino e aprendizado. Eles relatam que diversos alunos passaram a ter acesso a livros e materiais que antes não chegavam às localidades mais pobres. Também ressaltam que a tecnologia proporcionou aos professores das zonas rurais acesso à cursos antes indisponíveis nesses locais mais afastados [Gomar and Ravela 2012]. 


\section{Modelo Brasileiro: Projeto Um Computador por Aluno}

O Brasil possui mais de 200 milhões de habitantes e ocupa um espaço territorial maior que 8 milhões de $\mathrm{km}^{2}$ [IBGE 2013]. São mais de 39 milhões de estudantes entre o nível pré-escolar até o ensino médio [INEP 2014].

Por ser um país extenso, cuja população vive diferentes realidades econômicas, sociais e culturais e a complexidade para prover boa infraestrutura de acesso à internet em todo o país, o Projeto Um Computador por Aluno (UCA) não teve como objetivo primário levar os laptops para todos os alunos em todo o Brasil. Seu propósito foi desenvolver práticas pedagógicas que se apoiassem no uso da TIC para aprimorar o processo de ensino e aprendizado. A formação dos professores e experiências acadêmicas de práticas pedagógicas para aplicação de TIC na educação eram a base do Projeto [Marinho 2015].

O UCA nasceu de uma série de discussões e articulações entre o Governo Federal e diferentes pessoas ligadas ao meio acadêmico. O Projeto começou em 2007 e foi dividido em duas grandes fases. A primeira fase durou três anos e foi batizada de Pré-Piloto. Nesta fase, Os alunos e professores de cinco escolas, cada uma localizada em um dos municípios de Porto Alegre (RS), São Paulo (SP), Piraí (RJ), Palmas (TO) e Brasília (DF), receberam os 1.390 laptops doados pela Intel, Encore e OLPC. A finalidade dessa fase inicial era realizar testes e experimentações pedagógicas com o intuito de compreender como a inserção da informática afetaria o processo de ensino e aprendizagem de alunos e professores [SEED 2009].

Em 2010 teve inicio a segunda fase (Piloto) que procurou expandir a experiência pedagógica para mais de 300 escolas em todos os estados da federação. O Ministério da Educação adquiriu mais de 150 mil laptops através do Fundo Nacional de Desenvolvimento da Educação (FNDE) [Câmara dos Deputados 2010, Marinho 2015].

No mesmo ano, o Projeto UCA-Total foi lançado nos municípios de Tiradentes (MG), Santa Cecília do Pavão (PR), Terenos (MS), Barra dos Coqueiros (CE) e São João da Ponta (PA). Esse projeto levou laptops e internet para os mais de 10 mil alunos e professores de todas as escolas públicas do ensino fundamental e médio desses municípios. Posteriormente, o município de Caetés (PE) foi incluído no projeto [Lavinas and Veiga 2013].

Ainda em 2010, foi criado o Programa Um Computador por Aluno (PROUCA). Ao contrário do Projeto UCA, o Programa possui dotação orçamentária própria e seu principal objetivo é mais próximo à proposta inicial do OLPC, adquirir e distribuir laptops e internet para todos os alunos do ensino fundamental e médio do país [Brasil 2010].

\subsection{Quantidade de Alunos e Professores atendidos}

A Subseção 4.1 apresentou a proporção de alunos e professores contemplados com computadores no Uruguai através do Plano Ceibal. Não é adequado comparar esses números com o do Projeto UCA, uma vez que no projeto uruguaio o objetivo inicial era entregar um computador para cada aluno do país. No Brasil, o foco foi na a prática pedagógica e experiência de novos métodos de ensino e aprendizagem. De qualquer forma, a revisão da literatura e de sites oficiais não nos permitiu chegar a números confiáveis. A entrega dos computadores, e a disponibilidade de acesso à internet, variou de região para região. Em alguns locais, dado o perigo de violência e possível roubo dos computadores, as escolas em conjunto com as famílias decidiram se os computadores poderiam ou não ser levados para casa [Lavinas and Veiga 2013, Marinho 2015]. 
Em 2013, a quantidade de computadores adquiridos pelo Governo Federal e governos locais superava 387 mil laptops [Borges 2013]. Entretanto, as fontes desses números são escassas e difíceis de serem confirmadas. Também não foi possível determinar quantos desses computadores ainda estão em funcionamento.

\subsection{Formação dos Professores}

A formação dos professores para trabalhar com as tecnologias disponibilizadas pelo Projeto UCA aconteceram no formato presencial e EAD. Ela visou atender a três dimensões: 1) domínio dos recursos tecnológicos para o uso do sistema Linux Educacional e de aplicativos existentes nos laptops educacionais; 2) uso dos laptops nos processos de ensinar e aprender, bem como na gestão de tempos, espaços e relações entre os protagonistas da escola, do sistema de ensino e da comunidade externa; 3) busca da articulação de teorias educacionais que permitam compreender criticamente os usos em diferentes contextos e reconstruir as práticas pedagógicas e de gestão da sala de aula e da escola [SEED 2009].

$\mathrm{Na}$ época, o MEC disponibilizou um modelo de formação permitindo que cada universidade local, ou Núcleo de Tecnologia Educacional (NTE), local adaptassem o curso para atender às necessidades das escolas participantes do UCA [SEED 2009].

Além dos professores em exercício, as equipes administrativas da escola (diretores, vice-diretores e coordenadores) e professores temporariamente alocados nas secretarias de edução municipais e estaduais também passaram pelos cursos de capacitação [SEED 2009]. Segundo Marinho (2015), essas pessoas deveriam ser formadas, pois futuramente elas teriam que lidar com as TICs quando retornassem para às salas de aulas.

Cabe ressaltar que na presente pesquisa não foi possível encontrar dados confiáveis sobre o número de pessoas formadas pelo Projeto UCA e nem pelo UCA-Total.

\subsection{Meio de Integração}

Enquanto ativo, o Projeto UCA possuía o Portal UCA como seu principal meio de integração. Entretanto, durante o período da escrita desse trabalho, o site encontrou-se fora do ar.

Também durante a produção desse trabalho, foi possível perceber que os sites do Ministério da Educação sobre o Programa UCA (PROUCA) estão desatualizados, com informações que datam de 2012.

\subsection{Envolvimento da Sociedade}

As universidades estiveram particularmente envolvidas com o Projeto, tanto na formação dos professores quanto no desenvolvimento de softwares e, principalmente, propondo práticas pedagógicas apoiadas nos computadores disponibilizados pelo UCA [Marinho 2015].

Na pesquisa não foi possível identificar iniciativas significativas oriundas da sociedade civil organizada.

\subsection{Resultados alcançados}

Assim como ocorrido com o Plano Ceibal (Subseção 4.5), não há unanimidade com relação aos resultados do UCA. Lavinas e Veigas (2013) escrevem que o UCA-TOTAL foi 
marcado por um baixo nível de aproveitamento e um padrão de funcionamento bastante divergente em cada município. Segundo elas, isso reflete as forças e debilidades locais e impede que o potencial do projeto seja completamente aproveitado. Elas destacam que os custos tecnológicos (aquisição, entrega e substituição de computadores e disponibilização de internet) são elevados, principalmente em cidades afastadas dos centros urbanos. Entretanto, elas ressaltam que o Projeto também é capaz de gerar alguns resultados positivos, pois alunos das classes sociais mais pobres descobriram a informática e a internet e passaram a dominá-las.

Cysneiros et al. (2011) escrevem que alguns componentes de infraestrutura influenciaram negativamente o Projeto. Segundo os autores, esses componentes seriam: A falta de infraestrutura das escolas para lidar com as TIC (conexão com a internet em velocidades baixas, pouco espaço físico para utilização e guarda dos laptops e falta de instalações elétricas para recarga); A violência e o medo dos alunos serem vítimas de crimes por estarem portando um equipamento móvel; e a falta de habilidade dos professores em lidar com as TICs.

Para Marinho (2015), o Projeto UCA acaba sendo avaliado pela perspectiva da proposta original do OLPC. O professor fala que essa não é a análise mais adequada, pois o UCA não teve como objetivo principal entregar um computador para cada criança no Brasil. A proposta de inovação pedagógica com concurso de tecnologia móvel, inclusive toda a formação dos professores, tinha ênfase na formação do mesmo para trabalhar com tecnologias. Segundo o professor, é possível analisar a implementação brasileira e outras que adotam o OLPC, porém não devemos compará-las, pois o Brasil- pelo seu tamanho, sua diversidade e sua realidade sócio-econômica-cultural - apresenta uma complexidade muito maior do que países como o Uruguai. O professor conclui que o projeto cumpriu seu objetivo de gerar uma ampla gama de estudos, análises, experiências e práticas de aplicações de TIC para aprimorar o processo pedagógico de ensino e aprendizagem.

Outros autores corroboram com Marinho e avaliam aspectos sobre a motivação e participação dos professores e alunos para utilizar as TICs em sala de aula. Segundo eles, o Projeto UCA foi capaz de influenciar positivamente alguns desses aspectos qualitativos [da Silva and Susin 2012, Alvarez et al. 2015, Universidade Federal do Ceará 2015].

O Projeto UCA e o UCA-Total, aparentemente, foram encerrados em 2013. O PROUCA ainda existe e há algumas universidades empenhadas na formação de professores que atuam em escolas que são atendidas pelo Programa [Universidade Federal do Ceará 2015]. Entretanto, é possível perceber que a acadêmia não tem se envolvido com o PROUCA de forma tão intensa quanto do início do UCA.

\section{Conclusões}

Nesse artigo foram apresentados alguns conceitos sobre a proposta do OLPC e suas premissas básicas. Também foram apresentadas as implementações da mesma no Brasil, através do Projeto UCA e no Uruguai, através do Plano Ceibal. Buscamos evidenciar o que deu certo e quais os desafios enfrentados em cada implementação, buscando contribuir com futuras propostas do uso das TICs para a educação.

Analisando os dados aqui expostos, qualquer comparação entre os projetos uruguaio e brasileiro deve ser feita com cuidado. Embora ambos sejam originados da mesma 
proposta, eles possuem objetivos e estão inseridos em contextos muito distintos. O Ceibal teve como objetivo a universalização do acesso às TICs, enquanto o UCA buscou desenvolver propostas pedagógicas para alterar o processo de ensino e aprendizagem nas escolas brasileiras.

O plano uruguaio tem a vantagem de ser aplicado em um país geograficamente pequeno, altamente alfabetizado e com a população de cerca de 3,4 milhões de pessoas. Já o UCA viveu realidade oposta. A dimensão territorial do Brasil e a falta de infraestrutura apresentam desafios logísticos para entrega, manutenção, substituição de equipamentos, e conexão com a internet. Além disso, a violência nas grandes cidades brasileiras é outra barreira para utilização de TICs na educação, principalmente se esse uso se der através de equipamentos móveis transportados pelos alunos.

Outro ponto destacado foi a relação de alunos e professores atendidos pelas implementações. É possível concluir que o Plano Ceibal está sendo bem sucedido no seu objetivo inicial de universalizar o acesso às tecnologias e as informações, pois uma parcela significativa da população já faz uso das TICs disponibilizadas pelo Plano. Já O UCA carece de informações quantitativas. Os dados encontrados durante a pesquisa estão desatualizados e não são provenientes de uma fonte de informação oficial do governo. Entretanto, mais uma vez ressaltamos que o objetivo primário do UCA não era entregar um computador na mão de cada aluno, logo não há expectativa de que esse Projeto tenha alcançado números próximos ao do Ceibal.

Também foram apresentados alguns dados sobre a formação dos professores para o uso das TICs na educação. Percebe-se que essa é a atividade essencial para o sucesso desse tipo de empreitada. Tanto no Ceibal quanto no UCA, são inúmeros os trabalhos que escrevem sobre a necessidade de formação e treinamento contínuo de professores. Diversos autores afirmam que os resultados obtidos com a inclusão de TICs sem a formação dos docentes acabam ficando muito aquém do potencial.

[...] o equipamento evolui com o tempo, ontem eram os netbooks, hoje são tablets e celulares e amanha será outra coisa. O que importa é que o professor seja capaz de adotar esses recursos em sua prática pedagógica e fazer com que os alunos aprendam a aprender. [Marinho 2015]

Ainda sobre a formação dos professores, é possível concluir que a formação presencial acontece em ambos os países, porém o formato EAD tem se mostrado altamente atrativo para disseminar o conhecimento. Ele tende a ter um custo menor que a formação presencial e consegue alcançar os professores em áreas afastadas dos grandes centros urbanos.

Além da formação, ter um meio de integração entre os diferentes interessados é de grande relevância. O Portal Ceibal é o repositório de informações, links e contatos sobre o Plano. Quase todas as informações estatísticas e históricas sobre o Ceibal constantes nesse trabalho vieram dele ou de links apontados por ele.

Mesmo formando os professores para trabalhar com as TICs e tendo um meio para compartilhar informações, é interessante, porém difícil, envolver a sociedade no Projeto. O Ceibal teve êxito nessa iniciativa e é possível perceber que isso contribui positivamente com o Plano. As redes de apoio ajudam o país a vencer alguns desafios que o governo sozinho teria dificuldade de superar. 
Por fim, é fundamental também que essas implementações façam parte de uma política pública que as habilite a gerar bons resultados a longo prazo. O UCA sofre por não fazer parte de uma política de Estado, fortemente apoiada pelo governo e sociedade.

\section{Agradecimentos}

Gostaríamos de expressar nossos sinceros agradecimentos ao Professor Simão Pedro Marinho (PUC-Minas) por toda a contribuição e atenção que nos deu para o desenvolvimento desse trabalho.

\section{Referências}

Alvarez, C. S., Moll, J., and Souza, D. O. (2015). O Laptop na Educação: aportes sobre o Projeto Piloto UCA Brasil. Revista e-curriculum, 13:28 - 60 .

Alves, R. M., Sampaio, F., and da Fonseca Elia, M. (2015). Duinoblocks: Desenho e implementação de um ambiente de programação visual para robótica educacional. Revista Brasileira de Informática na Educação, 22(03):126.

Borges, P. (2013). Vitrine de lula, programa um computador por aluno só chegou a $2 \%$ dos estudantes. http://ultimosegundo.ig.com.br/educacao/2013-08-20/vitrine-de-lulaprograma-um-computador-por-aluno-so- chegou-a-2-dos-estudantes.html. Acessado em abril de 2015.

Brasil (2010). Decreto Presidencial 7.243/2010 regulamenta o Programa Um Computador por Aluno (PROUCA) e o Regime Especial de Aquisição de Computadores para uso Educacional (RECOMPE). Palácio do Planalto.

Canal, M. and Almeida, L. (2010). O desing do laptop educacional do olpc: uma avaliação usando as leis da simplicidade. Brasil: Instituto de Computação da Universidade Estadual de Campinas.

CIA (2015). The world factbook. https://www.cia.gov/library/ publications/the-world-factbook/. Acessado em abril de 2015.

Cysneiros, P. G., Carvalho, A. B. G., and Panerai, T. (2011). O programa uca na visão de professores multiplicadores. Anais do XXII SBIE-XVII WIE Aracaju, pages 19461952.

Câmara dos Deputados (2010). Um computador por aluno: a experiência brasileira. Technical report.

da Silva, C. O. and Susin, L. (2012). Aprendizagens e vivência virtuais: Uma análise sobre o projeto uca em aulas de ciências. In IX ANPED Sul - Seminário de Pesquisa em Educação da Região Sul, pages 1-15.

de Melo, G., Machado, A., and Miranda, A. (2014). The Impact of a One Laptop per Child Program on Learning: Evidence from Uruguay. Technical Report 2014-22, Banco de México.

Ferrando, M., Machado, A., Perazzo, I., and Vernengo, A. (2010). Una primera evaluación de los efectos del Plan Ceibal en base a datos de panel. Technical report, Instituto de Economía de la FCEydeA.

Gil, A. C. (1999). Métodos e técnicas de pesquisa social. Atlas, $5^{\mathrm{a}}$ edition. 
CBIE-LACLO 2015

Anais do XXI Workshop de Informática na Escola (WIE 2015)

Gomar, G. P. and Ravela, P. (2012). Impactos del Plan Ceibal en las práticas de enseñanza en las aulas de primaria. PhD thesis, Instituto de Evaluación Educativa, Facultad de Ciencias Humanas, Universidad Catolica.

IBGE (2013). Pesquisa nacional de amostras por domicílio (pnad). Acessado em abril de 2015.

INEP (2014). Censo escolar da educação básica de 2014. http : / / download . inep. gov.br/educacao_basica/censo_escolar/resultado/2014/. Acessado em maio de 2015.

Kraemer, K. L., Dedrick, J., and Sharma, P. (2009). One laptop per child: vision vs. reality. Communications of the ACM, 52(6):66-73.

Lavinas, L. and Veiga, A. (2013). Desafios do modelo brasileiro de inclusão digital pela escola. Cadernos de Pesquisa, 43:542 - 569.

Marandino, J. and Wunnava, P. V. (2014). The effect of access to information and communication technology on household labor income: Evidence from one laptop per child in uruguay. (8415).

Marinho, S. P. (2015). Projeto uca. Depoimento. [12/05/2015]. Entrevista concedida aos autores do artigo.

MEC (2013). Anuario Estadístico de educación. Technical report, Dirección de Educación. Área de Investigación y Estadística.

Melo, G. d., Machado, A., Miranda, A., and Viera, M. (2013). Profundizando en los efectos del Plan Ceibal. Technical report, Instituto de Economía - FCEyA - UdelaR, Montevideo, Uruguay.

Nugroho, D. and Lonsdale, M. (2010). Evaluation of olpc programs globally: a literature review. Melbourne, Australia: Australian Council of Educational Research. Mimeographed document.

Papert, S. (1993). The Children's Machine: Rethinking School in the Age of the Computer. BasicBooks.

Plan Ceibal (2015). Historia. http: / /www. ceibal. edu.uy/. Acessado em abril de 2015.

Rivoir, A. L. and Lamschtein, S. (2012). Cinco años del Plan Ceibal: algo más que una computadora para cada niño. Technical Report 2014-22, Unicef.

Sampaio, F. F. and da Fonseca Elia, M. (2012). Projeto um computador por aluno: pesquisas e perspectivas. NCE/UFRJ, Rio de Janeiro, RJ.

SEED (2009). Projeto "Um Computador por Aluno" - Formação Brasil: Projeto, planejamento das ações / cursos. Ministério da Educação.

Universidade Federal do Ceará (2015). Prouca sobral recebe novos cursistas em sua formação. http://blogs.virtual.ufc.br/uca-ce2/ ?p=1703. Acessado em maio de 2015. 